\title{
GOVERNANÇAS E MEDIAÇÕES ALGORÍTMICAS DA PLATAFORMA YOUTUBE DURANTE A PANDEMIA DE COVID-19
}

\author{
Gregório de Almeida Fonseca ${ }^{1}$ \\ Carlos Frederico de Brito d'Andréa
}

Resumo:

0 presente artigo explora como a governança e as mediações algorítmicas da plataforma YouTube se modificaram durante os primeiros meses da pandemia de COVID-19. Para compreender as políticas de moderação, de monetização e de recomendação de conteúdos considerados confiáveis ou desinformativos, o trabalho concentra-se em duas frentes de análise: a exploração dos documentos publicados pelo YouTube e de dados sobre os vídeos mais 'relevantes' sobre "vacina" exibidos antes e depois do início da pandemia. Dentre os resultados, destacam-se um aumento nos esforços institucionais da plataforma para moderar a circulação de vídeos desinformativos e uma maior "relevância" atribuída a vídeos recentes sobre o tema.

Palavras-chave: estudos de plataforma; COVID-19; métodos digitais; governança; YouTube.

\section{Abstract:}

This article explores how the governance and algorithmic mediations of the YouTube platform changed during the first months of the COVID-19 pandemic. To understand the policies of moderation, monetization and recommendation of content considered reliable or disinformative. The work focuses on two fronts of analysis: the exploration the documents published by YouTube and data on the most relevant "vaccine" videos displayed before and after the start of the pandemic. Among the results, we highlight an increase in the institutional efforts to moderate the circulation of disinformative videos and a greater "relevance" attributed to recent videos on the topic.

Keywords: platform studies; COVID-19; digital methods; governance; YouTube.

\section{Introdução}

Os conteúdos relacionados à disseminação da COVID-19³ no mundo, caracterizada como pandemia pela Organização Mundial de Saúde em março de 2020, ${ }^{4}$ ocuparam as plataformas online que utilizamos. Dado o distanciamento social praticado para conter o espalhamento do vírus Sars-CoV- $2,{ }^{5}$ mídias sociais como Twitter, Facebook e YouTube passaram a ter um papel ainda mais importante para a comunicação das pessoas ao

\footnotetext{
1 Doutorando em Comunicação Social na Universidade Federal de Minas Gerais (UFMG), mestre em Engenharia pelo Instituto Tecnológico de Aeronáutica (ITA) e bacharel em Engenharia Elétrica pela UFMG.

2 Professor do Programa de Pós-Graduação em Comunicação Social da UFMG e coordenador do grupo de pesquisa R-EST - estudos redes sociotécnicas.

3 COVID-19 é a sigla em inglês para "coronavírus disease 2019", cuja tradução para o português é "doença do coronavírus 2019". A sigla COVID-19 também é usada para se referir à doença em ambos os idiomas.

$4 \mathrm{https}$ // / www.who.int/docs/default-source/coronaviruse/situation-reports/20200311-sitrep-51-covid19.pdf?sfvrsn=1ba62e57_10

5 Sars-CoV-2 é o nome do vírus causador da COVID-19.
} 
conectar indivíduos fisicamente distantes por longos períodos e ao mediar o conteúdo distribuído pelas próprias plataformas. Durante o período de incertezas da pandemia, informações baseadas em evidências científicas e conteúdos considerados desinformativos vêm disputando espaço e visibilidade nas plataformas, as quais tentam se adequar às novas circunstâncias fazendo ajustes normativos, econômicos e computacionais.

0 presente artigo busca explorar como a governança e as mediações algorítmicas da plataforma YouTube se modificaram durante os primeiros meses da pandemia de COVID-19 (março, mês em que a doença foi caracterizada como pandemia pela Organização Mundial de Saúde ${ }^{6}$ a junho de 2020), principalmente no que tange à publicação e à circulação de conteúdos considerados confiáveis ou desinformativos. A perspectiva utilizada para se abordar o tema tem como referência as mediações tecnopolíticas exercidas pela plataforma YouTube. Ao se referirem aos Estudos de Plataformas, Plantin et al. (2016, p. 293, tradução nossa) destacam que eles "capturam como a comunicação e a expressão são ativadas e restritas pelos novos sistemas digitais e novas mídias". ${ }^{7}$ É importante observar que, nessa abordagem, as plataformas são tomadas como um fator constituidor do fenômeno empírico que está sendo estudado. Nos Estudos de Plataformas:

devemos procurar entender tanto o modo como algoritmos, recursos
tecnogramaticais (curtir, compartilhar etc.), políticas de governança
(como os termos de uso) etc. moldam as práticas e as percepções dos
usuários, quanto as apropriações criativas, táticas e coletivas que recriam,
cotidianamente, as plataformas (D’ANDRÉA, 2020).

O YouTube é uma plataforma de grande relevância no ecossistema de informação no Brasil. Newman et al (2019) produziram um relatório baseado em um questionário online onde apresentam o resultado de que $80 \%$ dos brasileiros se declaram usuários do YouTube e $42 \%$ utilizam a plataforma para consumir notícias. Dados do Statista (2020), que classificam a popularidade das plataformas ao redor do mundo, apontam o YouTube como a segunda maior em número de usuários, totalizando dois bilhões de usuários ativos a cada mês.

Em um ambiente digital com uma participação tão intensa, mesmo uma pequena porcentagem de usuários pode significar um alcance de milhares ou mesmo milhões de

6 https://www.who.int/docs/default-source/coronaviruse/situation-reports/20200311-sitrep-51-covid19.pdf?sfvrsn=1ba62e57_10

7 No original: "platform studies captures how communication and expression are both enabled and constrained by new digital systems and new media"

// REVISTA DISPOSITIVA, v. 9, n. 16, p. 6-26 - ago/dez (2020) // 
pessoas. O YouTube (2019c), sem apresentar os dados brutos, chegou a afirmar que cerca de $1 \%$ de todo o conteúdo assistido na plataforma é considerado "limítrofe" 8 (que chega perto de violar as diretrizes da plataforma) ou pode ser tomado como desinformação prejudicial. No contexto da pandemia, uma pesquisa de Li et al. (2020), observando uma amostra de 69 vídeos, identificou que 27,5\% dos vídeos mais vistos sobre COVID-19 em língua inglesa continham desinformação. Assim, como discute Gillespie (2018), a adoção de medidas de governança como a moderação de conteúdo é central para as plataformas.

O presente artigo explora a transformação da atuação do YouTube durante os quatro primeiros meses da pandemia por meio de duas frentes de análise. A primeira tem como foco a atualização de políticas e de diretrizes com base em documentos publicados pelo YouTube, como termos de serviço e políticas, bem como em outros materiais especializados, como notícias publicadas na imprensa. Nesta frente de análise, a governança da plataforma é discutida com foco na moderação e na monetização de conteúdos.

$\mathrm{Na}$ segunda frente, foram explorados dados fornecidos via API do YouTube utilizando-se a ferramenta YouTube Data Tools (RIEDER et al, 2018). 0 foco aqui volta-se para as recomendações algorítmicas feitas pela plataforma por meio de sua busca interna. Foram monitoradas, antes e no início da pandemia, as variações nos vídeos mais "relevantes" sobre o tema "vacina", segundo o YouTube. Nossa referência aqui são “métodos digitais” que, na definição de Venturini et al. (2018, p. 4195, tradução nossa), lidam com "o reaproveitamento das inscrições geradas pela mídia digital para o estudo dos fenômenos coletivos". 9 Pela abordagem dos métodos digitais, as especificidades sociotécnicas da plataforma em que está inserido o objeto de pesquisa são essenciais para a definição de como, e em que medida, é possível estudar os meios digitais - ou, no caso desta pesquisa, a plataforma YouTube.

Iniciamos o artigo com uma breve caracterização do YouTube como uma plataforma e, na sequência, apresentamos a análise empírica fundamentada nas duas frentes de análise mencionadas acima. Nas reflexões finais, discutimos as principais descobertas do estudo, entre as quais um aumento nos esforços para evitar a publicação de vídeos desinformativos (relativo à governança) e um significativo aumento de vídeos muito

8 "Borderline", no original em inglês.

9 No original: "Digital Methods can be defined as the repurposing of the inscriptions generated by digital media for the study of collective phenomena." 
recentes (publicados há menos de cinco dias) dentre os mais "relevantes" com o tema "vacina” (mediações algorítmicas).

\section{YouTube como plataforma}

As plataformas como o YouTube veem se transformando e se tornando mais complexas ao longo dos anos, sendo importante observá-las a partir suas modificações e suas instabilidades. Em alinhamento com os Estudos de Plataformas, Burgess (2018, p. 10, tradução nossa) aponta que "embora o YouTube por muitos anos tenha conseguido manter uma imagem de si mesma como uma plataforma neutra para compartilhar conteúdo, em vez de um provedor de conteúdo, não há mais como negar que ele faz as duas coisas”. ${ }^{10}$

Assim, a plataforma medeia e modera o conteúdo que nela circula de acordo com as regras que ele mesmo define. Como forma ativa de moderação de conteúdo, o YouTube remove conteúdos que não se adequam às suas diretrizes. A remoção pode dar-se tanto por meio de moderadores humanos quanto por ferramentas automatizadas. Ressaltando as articulações sociomateriais da moderação, Roberts (2020, tradução nossa) ressalta que “é extremamente difícil para esses sistemas trabalhar sem a supervisão humana ou na ausência de humanos verificando e fazendo remoções manuais." 11

Como reflexo da pandemia de COVID-19, o YouTube optou por reduzir o número de funcionários trabalhando em seu escritório e declarou que aumentou a utilização de tecnologias de automatização para realizar parte do trabalho que anteriormente era feito por moderadores humanos a partir de maio. (YOUTUBE, 2020b). Por sua vez, o "painel de verificação de fatos", um recurso que existia desde 2019 no Brasil (GOOGLE, 2019), foi expandido para os Estados Unidos em 2020 em função da pandemia (YOUTUBE, 2020e). Esse painel exibe checagens realizadas por editores qualificados externos ao YouTube quando são realizadas pesquisas relacionadas a tópicos propensos a "teorias da conspiração em andamento" (GOOGLE, 2019). É uma forma de alertar o usuário priorizando a exibição de uma informação verificada.

10 No original: "While YouTube for many years managed to maintain an image of itself as a neutral platform for sharing content, rather than a content provider, there can no longer be any denying that it does both". 11 No original: “(...) is exceedingly difficult for those systems to work without human oversight or in the absence of humans vetting and doing manual removals, too". 
Análise documental da plataforma

Nesta frente de análise, empreendemos uma análise documental baseada em documentos e comunicados divulgados pelo YouTube, tais como suas políticas, diretrizes e ações antes e durante a pandemia, bem como em algumas notícias publicadas na internet envolvendo a plataforma e sua resposta à pandemia. Os eixos de análise tratados nesta seção são os termos de serviço e políticas da plataforma, que englobam os seus principais aspectos de governança; a monetização, cujas mudanças influenciam diretamente a estratégia de publicação de vídeos por produtores de conteúdo e a moderação da desinformação, como forma de mediação do que circula no YouTube. Combinados, esses eixos de análise possibilitam uma análise aprofundada da governança e das mediações algorítmicas da plataforma.

\section{Termos de serviço e políticas da plataforma}

Os termos de serviço, de acordo com Gillespie (2018, p. 45, tradução nossa), são "um contrato que especifica os termos sob os quais o usuário e a plataforma interagem, as obrigações que os usuários devem aceitar como condição de sua participação e os meios adequados para resolver uma disputa". ${ }^{12}$ Como principais características do documento, Gillespie (2018) enuncia os principais conteúdos que fazem parte do texto: conteúdo e comportamento apropriados, responsabilidades, propriedade intelectual e questões de arbitragem.

Desde o primeiro momento em que a COVID-19 passou a ser considerada uma pandemia, em março de 2020, os termos de serviço vigentes no YouTube estão na mesma versão, que foi publicada em 10 de dezembro de 2019 (YOUTUBE, 2019b). A plataforma se posiciona de forma a limitar a sua responsabilidade em relação ao conteúdo que é disponibilizado pelos usuários. Até o momento em que os resultados da presente pesquisa foram compilados (julho de 2020), o seguinte trecho destacava essa posição:

12 No original: "a contract that spells out the terms under which user and platform interact, the obligations users must accept as a condition of their participation, and the proper means of resolving a dispute should one arise". 


\begin{abstract}
"Limitação de responsabilidade
EXCETO SE EXIGIDO PELA LEGISLAÇÃO APLICÁVEL, O YOUTUBE, SUAS AFILIADAS, DIRETORES, CONSELHEIROS, FUNCIONÁRIOS E AGENTES NÃO SERÃO RESPONSÁVEIS POR LUCROS CESSANTES, RECEITAS, OPORTUNIDADES DE NEGÓCIOS, FUNDOS DE COMÉRCIO OU ECONOMIAS ANTECIPADAS; PERDA OU CORRUPÇÃO DE DADOS; PERDA INDIRETA OU CONSEQUENTE; DANOS PUNITIVOS CAUSADOS POR:

(...)

6. QUALQUER CONTEÚDO ENVIADO PELO YOUTUBE OU POR UM USUÁRIO, INCLUINDO O USO QUE VOCÊ FAZ DO CONTEÚDO" (YOUTUBE, 2019b)
\end{abstract}

Os termos de serviço subsidiam um discurso de ausência de responsabilidade da plataforma. Conforme Gillespie (2018, p. 46, tradução nossa), "é possível que (os termos de serviços) sejam escritos com o objetivo de evitar litígios futuros, geralmente indenizando a empresa o mais amplamente possível contra qualquer responsabilidade pelas ações dos usuários". ${ }^{13}$ Dessa forma, por mais que a plataforma tenha uma postura ativa de moderação de conteúdo, ela busca sempre evitar problemas legais.

Durante os primeiros meses da pandemia de COVID-19, no entanto, o YouTube parece ter ajustado suas políticas com o intuito de mediar mais efetivamente os conteúdos que nele circulam. A plataforma não atualizou o texto de seus termos de serviço, mas definiu ações e diretrizes em uma carta aos criadores de conteúdo (YOUTUBE, 2020c) e na página “Atualizações sobre o coronavírus 2019 (COVID-19)” (YOUTUBE, 2020b). No período entre 11 de março e 20 de maio de 2020, foram registradas vinte alterações nessa página ou, aproximadamente, uma atualização a cada três dias - o que reflete a necessidade de constante adaptação em um momento de incertezas. Por sua vez, entre 20 de maio e o início de julho de 2020, não foram registradas novas atualizações, o que indica que, ao menos em relação às políticas da plataforma, o YouTube chegou a um certo ponto de estabilidade.

\title{
Monetização
}

O YouTube é uma plataforma que recompensa financeiramente os seus criadores de conteúdo por meio de recursos de monetização dentro de um programa de parcerias que "permite que os criadores compartilhem a receita de publicidade gerada pelas

13 No original: "It is arguably written with an eye toward avoiding future litigation, often indemnifying the company as broadly as possible against any liability for users' actions." 
visualizações em seus vídeos”14 (BURGESS, 2018, p. 55, tradução nossa). 0 programa de parcerias possibilita ao criador de conteúdo que ganhe dinheiro com receita de publicidade, clubes dos canais, estante de produtos do canal, super chat e super stickers e receita do YouTube Premium (GOOGLE, 2020).

Em uma tentativa de minimizar a produção de conteúdo desinformativo, a primeira grande ação do YouTube foi bloquear a obtenção de receitas por meio de vídeos que tratavam de temas ligados à pandemia de COVID-19. 0 início dessa desmonetização foi identificado pela repórter Alexander (2020) em artigo publicado no The Verge em 4 de março de 2020. De acordo com a autora, o YouTube informou que a pandemia foi classificada como um "evento delicado", o que justificaria a desmonetização. Na página “Diretrizes de conteúdo adequado para publicidade", o termo "pandemia" não aparece citado nominalmente como algo "delicado" (YOUTUBE, 2020d), mas a lista (que inclui itens como “conteúdo de incitação ao ódio" e "conteúdo relacionado a armas de fogo") é apresentada como "incompleta", dando à plataforma um certo grau de liberdade para desabilitar a monetização.

A flexibilização na monetização dos vídeos relacionados à pandemia iniciou-se de forma pontual pouco mais de uma semana depois da publicação do The Verge. Em 11 de março de 2020, a monetização foi liberada para uma lista limitada de canais que incluía "parceiros de notícias" e criadores de conteúdo que utilizam corretamente as ferramentas de autocertificação. Essas ferramentas permitem ao criador classificar o material publicado quanto ao tipo de conteúdo e à monetização (YOUTUBE, 2020j). A preferência dada a este tipo de criadores de conteúdo é considerada por Perez (2020, tradução nossa), como algo "questionável, pois abre a monetização para qualquer criador que use o sistema de autoetiquetagem do YouTube, e não apenas organizações de notícias ou autoridades de saúde confiáveis". ${ }^{15}$ Vale lembrar, no entanto, o recurso não está disponível para todos os usuários.

No dia 16 de março de 2020, a lista de canais com monetização liberada foi ampliada. Além disso, foram dadas recomendações a serem seguidas pelos criadores de conteúdo antes de publicarem seu material: "faça uma checagem de fatos, leve em conta que esse evento é uma crise global em andamento e siga as diretrizes da comunidade e de conteúdo adequado para a publicidade". Como forma de proteção à sua força de trabalho,

14 No original: “(...) which allows creators to share in advertising revenue generated by views on their videos”. 15 No original: “(...) is a more questionable choice, as it opens up monetization to any creator using YouTube's self-labeling system, not just news organizations or trusted health authorities." 
- YouTube decidiu ainda aumentar a quantidade de vídeos moderados de forma automatizada e reduzir o número de moderadores humanos durante o período da pandemia (YOUTUBE, 2020h). Considerando esta decisão, nota-se que faz sentido que a autocertificação seja realizada em maior escala e que a escolha de um grupo específico de criadores de conteúdo busca limitar os potenciais erros que possam surgir como consequência dessa autonomia.

No dia 2 de abril de 2020, após cerca de um mês de restrições relativas à monetização, o YouTube optou por permitir que quaisquer canais pudessem gerar receitas em seus vídeos relacionados à pandemia desde que seguissem as políticas e diretrizes da plataforma. Na página “Atualização sobre a monetização no conteúdo relacionado à COVID-19" (YOUTUBE, 2020a), foram listados alguns exemplos de conteúdos que continuam sem a monetização: “filmagens angustiantes”, “desinformação médica” e "pegadinhas e desafios".

\section{Moderação da desinformação}

Desinformação é um termo utilizado para tratar de informações imprecisas ou enganosas (VOSOUGHI et al., 2018). Wardle et al. (2017) apresentam dois termos em inglês que podem ser traduzidos para o português como desinformação: misinformation e disinformation. De acordo com os autores, misinformation são informações falsas criadas sem a intenção de causar dano, prejuízo ou mal; disinformation são as informações falsas criadas com o intuito deliberado de causar dano a uma pessoa, grupo social, organização ou país. O Intervozes - Coletivo Brasil de Comunicação Social, também adotou o termo desinformação, buscando "ressaltar a intencionalidade na produção e na propagação de informações falsas, equivocadas ou descontextualizadas para provocar uma crise comunicacional e, assim, obter ganhos econômicos e/ou políticos." (MARTINS, 2020, l. 151).

Em momentos como a expansão do vírus causador da doença COVID-19, a desinformação pode se agravar. Zarocostas (2020, tradução nossa) define o que passou a ser chamado de infodemia pela Organização Mundial de Saúde: “(...) uma epidemia global de desinformação - que se espalha rapidamente pelas plataformas de mídia social e outros 
meios de comunicação". ${ }^{16}$ McAweeney (2020) ressalta que, em momentos de pânico e de incerteza, as pessoas tendem a consumir informação de baixa qualidade em uma intensidade e velocidade ainda maiores. Dessa forma, enquanto as convicções científicas ainda não estão estabilizadas, são complexas e/ou são de implementação não imediata, determinados grupos se apegam a soluções simplistas, imediatistas ou outros conteúdos desinformativos. A desinformação relacionada à pandemia de COVID-19, em geral, pode ser chamada de desinformação científica ou, dentro do contexto da pandemia, de desinformação médica. Ela se encaixa nessa classificação ao contradizer, refutar ou distorcer um consenso (ainda que momentâneo) da comunidade científica e das autoridades de saúde pública.

Nas primeiras semanas da pandemia, o YouTube baseou-se na sua "Política de conteúdo perigoso ou nocivo" (YOUTUBE, 2020f) para remover o conteúdo identificado com desinformação médica. De acordo com essa política, “curas e remédios milagrosos” não podem ser promovidos na plataforma. É um tipo de definição que abre margem à discussão e pode exigir análises técnicas detalhadas antes da remoção ou não de um vídeo da plataforma. Assim, não é surpresa que conteúdos sobre tratamentos alternativos sem evidência científica estejam muito presentes no YouTube (LEMOS, 2020).

Além disso, essa restrição não engloba todo tipo de desinformação relacionada à COVID-19, o que levou o YouTube a criar uma "Política de informações médicas incorretas relacionadas à COVID-19" (YOUTUBE, 2020g). Baseada nas "orientações da OMS e das autoridades locais de saúde sobre: tratamento; prevenção; diagnóstico; transmissão”, esta diretriz definiu de uma forma mais precisa o que a plataforma considera desinformação. 0 Quadro 1 sintetiza as definições de informações médicas consideradas incorretas bem como alguns dos exemplos utilizados pelo próprio YouTube em sua nova política.

16 No original: "a global epidemic of misinformation-spreading rapidly through social media platforms and other outlets". 
Quadro 1. Informações médicas incorretas

\begin{tabular}{|c|c|c|}
\hline Categoria & Definição & Exemplos \\
\hline Tratamento & $\begin{array}{l}\text { Desestimula alguém a procurar } \\
\text { tratamento médico ao } \\
\text { encorajar o uso de remédios } \\
\text { ou curas para o tratamento da } \\
\text { COVID-19. }\end{array}$ & $\begin{array}{l}\text { Conteúdo que incentiva o uso } \\
\text { de medicamentos caseiros } \\
\text { como substitutos do tratamento } \\
\text { médico adequado (consultar } \\
\text { um médico ou ir ao hospital). } \\
\text { Conteúdo que incentiva o uso } \\
\text { de orações ou rituais como } \\
\text { substitutos do tratamento } \\
\text { médico }\end{array}$ \\
\hline Prevenção & $\begin{array}{l}\text { Promove métodos de } \\
\text { prevenção que contradizem as } \\
\text { informações da OMS ou das } \\
\text { autoridades locais de saúde. }\end{array}$ & $\begin{array}{l}\text { Afirmações de que } \\
\text { determinadas pessoas têm } \\
\text { imunidade à COVID-19 devido } \\
\text { a raça ou nacionalidade. } \\
\text { Vídeos com alegações de que } \\
\text { as pessoas não serão } \\
\text { infectadas se evitarem comida } \\
\text { asiática }\end{array}$ \\
\hline Diagnóstico & $\begin{array}{l}\text { Promove métodos de } \\
\text { diagnóstico que contradizem } \\
\text { as informações da OMS ou das } \\
\text { autoridades locais de saúde. }\end{array}$ & $\begin{array}{l}\text { Conteúdo com afirmações de } \\
\text { que o ato de prender a } \\
\text { respiração pode ser usado } \\
\text { como teste de diagnóstico para } \\
\text { a COVID-19. }\end{array}$ \\
\hline Transmissão & $\begin{array}{l}\text { Promove informações de } \\
\text { transmissão que contradizem } \\
\text { as informações da OMS ou das } \\
\text { autoridades locais de saúde. }\end{array}$ & $\begin{array}{l}\text { Conteúdo com declarações de } \\
\text { que algum grupo ou indivíduo } \\
\text { têm imunidade garantida ao } \\
\text { vírus ou não podem transmiti- } \\
\text { lo. } \\
\text { Afirmações de que a COVID-19 } \\
\text { é causada pela radiação das } \\
\text { redes } 5 \mathrm{G} \text {. }\end{array}$ \\
\hline
\end{tabular}

Fonte: Elaborado pelos autores com base nas informações do YouTube (2020g).

Vale ressaltar que as diretrizes reproduzidas no Quadro 1 foram elaboradas pela plataforma e não necessariamente englobam todo tipo de desinformação sobre vacinas. 0 que YouTube apresenta é sua interpretação da realidade que, no mínimo, tensiona o conhecimento científico. Uma das classificações definidas por Fernandes et al . (2020) ao categorizar a desinformação sobre vacinas, por exemplo, é "As vacinas beneficiam as indústrias farmacêuticas", tema que sequer é citado pelo YouTube (2020g).

Desde que o YouTube passou a atuar de uma forma mais proativa em relação ao conteúdo sobre a COVID-19, há relatos de diversas remoções de vídeos contendo desinformação. Ventura (2020), por exemplo, registrou a remoção de conteúdo alegando a "farsa do coronavírus". O ideólogo Olavo de Carvalho, de acordo com Azevedo (2020), 
também teve um vídeo em que afirmava que o "coronavírus não mata" removido do YouTube pela própria plataforma. Além disso, Silva (2020) e Tilt (2020) identificaram a remoção de vídeos contendo teorias da conspiração sobre a pandemia, como aqueles que atribuíam a transmissão do vírus às redes de telefonia $5 \mathrm{G}$.

Embora haja relatos de remoção de vídeos, entretanto, a desinformação acerca da pandemia continua circulando no YouTube. Dias et al. (2020) destacam uma das principais estratégias dos canais que, mesmo em desacordo com os termos de serviço da plataforma, permanecem sendo monetizados: ao não utilizar palavras-chave relativas à pandemia, os vídeos conseguem contornar as restrições do processo automatizado de moderação.

Outra estratégia de monetização é a colaboração com outros canais, apontada por Ohlheiser (2020a). A autora narra exemplos de youtubers conspiracionistas que, mesmo tendo seus canais removidos, conseguiram levar seu discurso ao público por meio da participação em outros canais da plataforma. Ohlheiser (2020b) conclui que a remoção de conteúdo pode impulsionar ainda mais o ciclo de desinformação, pois os produtores de conteúdo podem se aproveitar de teorias de conspiração alegando que estão sendo silenciados. Além disso, a remoção “pode vir tarde demais”, quando a desinformação já se espalhou.

Mediações algorítmicas: vídeos mais "relevantes" sobre "vacina”

A segunda frente de análise do artigo concentra-se nas recomendações algorítmicas de vídeos feitas pela plataforma por meio de sua busca interna. Como mencionado na introdução, essa análise se apoiou na perspectiva dos métodos digitais (VENTURINI et al., 2018), que se apropria de inscrições produzidas pelos meios para compreender sua relação com os fenômenos sociais. Mais especificamente, nos inspiramos aqui na análise das “culturas de ranqueamento” do YouTube elaborada por Rieder et al. (2018).

Com o objetivo de acompanhar a presença do termo "vacina"17 no YouTube, foram extraídos dados semiestruturados por meio da versão $v 3^{18}$ da API desta plataforma no período de janeiro de 2019 a junho de 2020. No contexto da pesquisa de doutoramento à qual se vincula este artigo, a escolha do termo "vacina" fora feita, desde 2018, visando

17 A solução para a erradicação da pandemia de COVID-19 a médio ou longo prazo passa pelo desenvolvimento de uma vacina. Embora o isolamento social e as restrições de aglomerações sejam atitudes que possam diminuir a dispersão do vírus, parece ser um consenso que uma retomada das atividades cotidianas dependerá do desenvolvimento de uma vacina para que a situação se aproxime do que era antes do início da pandemia.

18 https://developers.google.com/youtube/v3/ 
acompanhar a presença do movimento antivacina na plataforma, bem como analisar como se dá a disseminação e debate de informações relacionadas ao termo "vacina" no YouTube. As extrações de dados foram feitas mensalmente, por meio da ferramenta YouTube Data Tools ${ }^{19}$ (YTDT), desenvolvida e mantida por Bernhard Rieder (RIEDER et al., 2018).

Para cada mês observado, de janeiro de 2019 a junho de 2020, uma lista com os dez vídeos mais relevantes foi elaborada. A Figura 1 apresenta a quantidade de vídeos com o termo "vacina” relacionados à COVID-19 que apareceram entre os dez mais relevantes ao longo do tempo. A primeira menção ao assunto surgiu em fevereiro de 2020, em apenas um vídeo. Nesse momento, ainda não havia casos confirmados da doença no Brasil. A primeira confirmação de COVID-19 no Brasil aconteceu no dia 26 de fevereiro de 2020, de acordo com o Ministério da Saúde (2020).

O mês seguinte apresentou uma mudança radical no conteúdo apresentado: em março de 2020, todos os dez vídeos entre os mais relevantes continham conteúdo relacionado à pandemia. Nos meses seguintes, esse número variou entre cinco a nove. A média resultante dos meses de março a junho de 2020 foi de $80 \%$ dos vídeos relacionados à COVID-19.

Figura 1. Quantidade de vídeos com o termo "vacina" relacionados à COVID-19 entre os dez mais "relevantes" no YouTube.

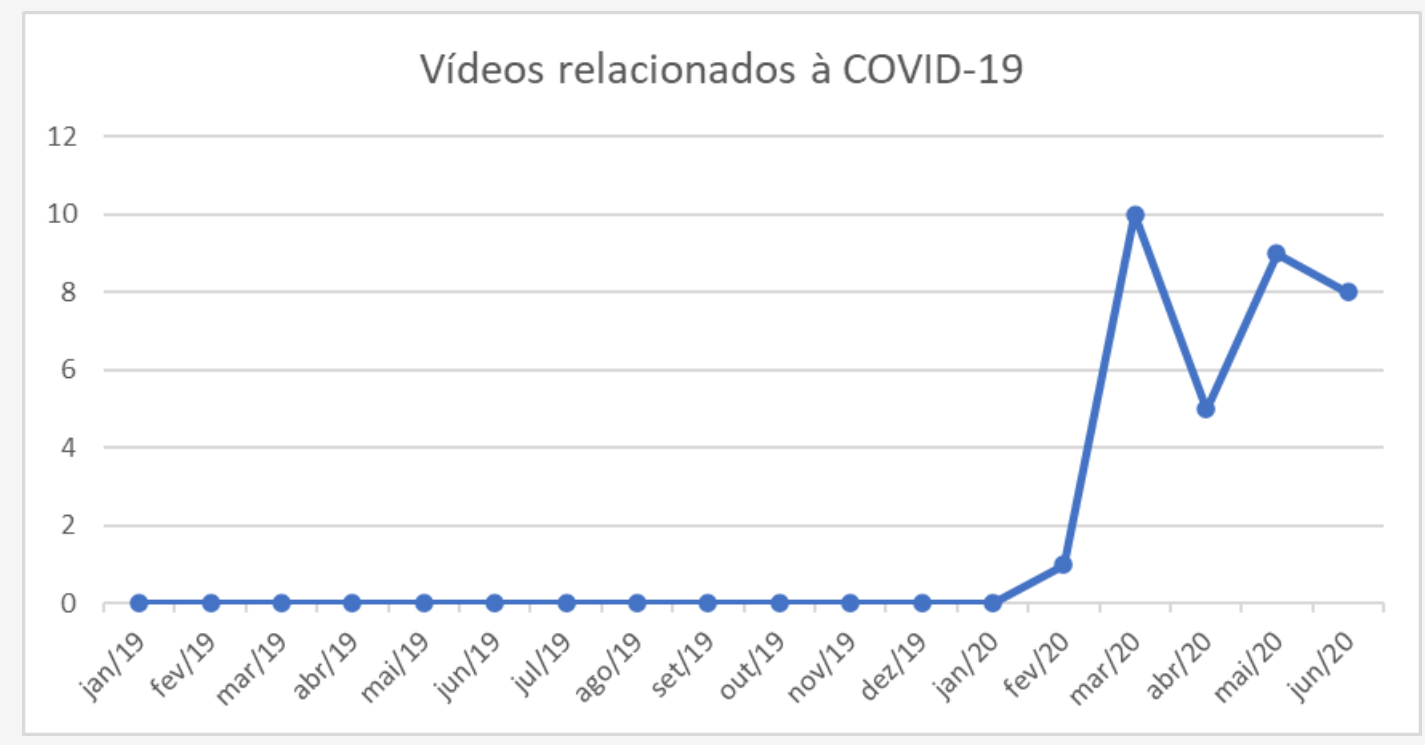

Fonte: elaborado pelos autores.

19 https: / / tools.digitalmethods.net/netvizz/youtube/ 
Outra grande mudança na lista de vídeos mais relevantes é o aumento da presença de conteúdo recente. A Figura 2Erro! Fonte de referência não encontrada. traça um comparativo da data de publicação dos dez vídeos mais relevantes de cada mês com o termo "vacina". Tendo como referência a data de extração de dados, foram definidas três categorias de análise: vídeos publicados há mais de um ano, vídeos publicados entre 6 dias e um ano, e vídeos publicados há menos de cinco dias. De janeiro a março de 2019, a grande maioria dos vídeos tinha sido postada há mais de um ano. De março a junho de 2020, os vídeos com menos de cinco dias de publicação representaram 62,5\% dos vídeos mais relevantes com o termo "vacina" apresentados pelo YouTube.

Figura 2. Comparativo da data de upload dos vídeos mais relevantes com o termo "vacina".

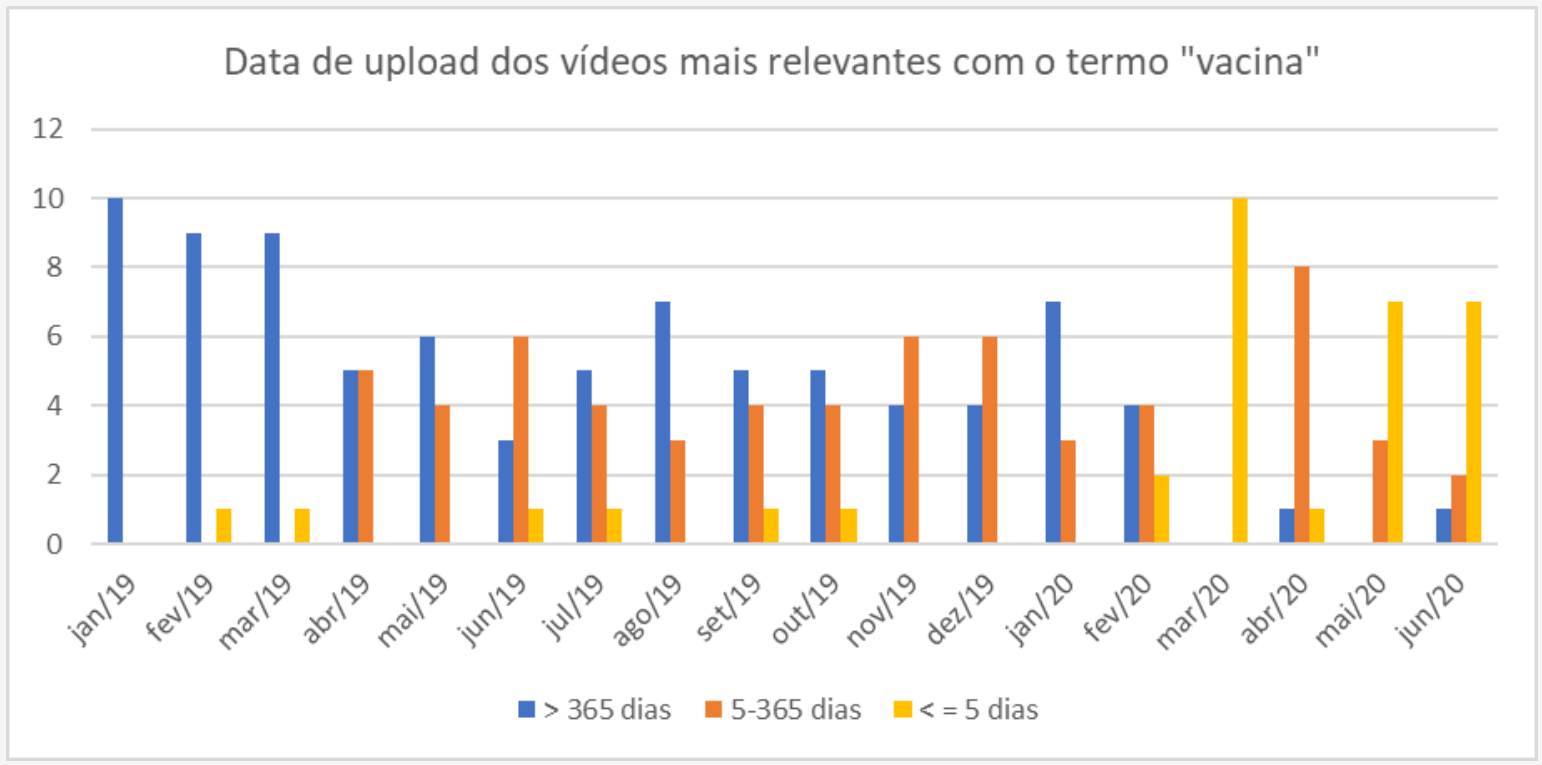

Fonte: elaborado pelos autores.

A mudança mais significativa no padrão aconteceu, assim como no gráfico da Figura 1 , no mês de março de 2020, momento em que a pandemia já apresentava casos confirmados de COVID-19 no Brasil. Nesse mês, todos os dez vídeos da lista de mais relevantes tinham sido publicados há menos de cinco dias, ${ }^{20}$ indicando uma mudança na

\footnotetext{
${ }^{20}$ Aqui é possível observar uma fragilidade do método de coleta de dados: ao realizar-se apenas uma extração de dados por mês, a representação pode ficar enviesada. Como vídeos mais recentes passaram a ser maioria entre os dez mais relevantes, se a coleta de dados acontecesse em períodos menores, outros vídeos teriam sido incluídos nos resultados.
} 
classificação do YouTube. No entanto, vale ressaltar que a priorização da apresentação de conteúdo mais recente não pode ser associada exclusivamente às mudanças algorítmicas da plataforma: as práticas dos usuários possivelmente mudaram durante a pandemia e a preocupação com a vacinação passou a ser um assunto de maior evidência pública no período. Além disso, produtores de conteúdo de alta visibilidade que não publicavam vídeos sobre o tema podem ter ganhado espaço ao tratá-lo, motivados pela pandemia.

É importante ressaltar que o termo "vacina" é um assunto relacionado à pandemia, mas, de forma alguma, engloba todos os vídeos relacionados à COVID-19 presentes no YouTube. A escolha por esse termo deu-se pela possibilidade de se fazer uma comparação com a situação anterior às mudanças e observar como a plataforma se adaptou durante o período. Além disso, por estarmos monitorando os dados históricos sobre "vacina" desde o início de 2019, foi possível observar as mudanças ao longo do tempo. Vale ainda ressaltar que a API do YouTube apresenta apenas os resultados de uma busca válidos no exato momento em que os dados são extraídos, ou seja, não seria possível simular uma busca pelo ordenamento em um período passado.

\section{Reflexões finais}

O presente artigo, por meio de análise documental e análise de dados obtidos via APIs, buscou explorar como a governança e as mediações algorítmicas da plataforma YouTube se modificaram durante os primeiros meses da pandemia de COVID-19, e quais foram os reflexos dessas mudanças na moderação e na circulação de conteúdos considerados confiáveis ou desinformativos.

Com base na pesquisa realizada, nota-se uma ação proativa da plataforma no sentido de limitar a disseminação de desinformação. A análise dos documentos providos pela plataforma indica uma adequação de seus termos de serviço e das políticas da plataforma para tratar de um assunto imprevisto (a pandemia de covid-19). Assim, as ações de restrição de monetização e exclusão de conteúdo anunciadas pelo YouTube, e identificadas em reportagens e pesquisas bibliográficas, apontam uma mediação mais efetiva da plataforma objetivando a minimização da circulação de conteúdo desinformativo.

Enquanto Gillespie (2018) destaca que as plataformas se colocam no direito, mas não na responsabilidade, de remover conteúdo, as ações do YouTube parecem ir um pouco 
além: nota-se um crescente senso de responsabilidade da plataforma sobre a informação que está circulando. As mudanças nas políticas, nas diretrizes e nos termos de serviço da plataforma, bem como as novas regras criadas no período, indicam uma maior preocupação com a credibilidade e confiabilidade do conteúdo que circula na plataforma. Por outro lado, a isenção de responsabilidade do YouTube pelo conteúdo publicado pelos usuários ainda está presente nos termos de serviço vigentes. A checagem da veracidade de fatos passou a ser mais estimulada, tanto por meio de recomendação do YouTube aos criadores de conteúdo quanto pela parceria da plataforma com checadores independentes.

As ações de limitação de monetização tiveram como objetivo inicial inibir a criação de conteúdos com desinformação que visassem prioritariamente ou apenas gerar receita. Enquanto ajustava seu processo de moderação, o YouTube adotou uma política bastante restritiva de monetização, mas aos poucos foi flexibilizando-a. Em parte, este recuo na política restritiva de monetização deve-se à pressão exercida por criadores de conteúdos avaliados como confiáveis, pois a receita paga pelo YouTube é o que financia (ao menos parcialmente) o custo de produção de muitos canais. Um exemplo de canal de divulgação científica que teve vídeos desmonetizados é o do biólogo Átila lamarino: em vídeo publicado no dia 15 de março de 2020, ${ }^{21}$ o cientista abriu ao público a possibilidade de tornarem-se assinantes pagos do canal como forma de obter receita e continuar produzindo conteúdo sobre a pandemia enquanto o YouTube não alterasse sua política de monetização. Posteriormente, os vídeos do biólogo voltaram a ser monetizados, e ele manteve o serviço de assinaturas como forma complementar de geração de receita.

Foi na mudança das mediações algorítmicas da plataforma que as adaptações da plataforma puderam ser observadas mais claramente. Na análise dos dados obtidos por meio da API do YouTube, identificou-se uma clara mudança do enfoque dos resultados de busca pelo termo "vacina", que deixaram de destacar vídeos mais antigos e visibilizaram vídeos muito recentes. Vale destacar que, em nenhum momento da pesquisa, conteúdos aparentemente desinformativos foram apresentados entre os mais "relevantes" associados ao termo "vacina", o que não significa que eles não continuem circulando e sendo acessados por outros meios. Assim, nota-se a priorização de conteúdo que apresente informações de acordo com as recomendações da OMS e autoridades de saúde, embora ainda sejam identificados pontos de fragilidade, como a circulação de vídeos contendo desinformação.

21 Por que o CORONAVÍRUS pode parar a sua vida? \#FiqueEmCasa. Disponível em: https: / /www.youtube.com/watch?v=Y10vCOXxtds 
Quando um usuário tem acesso a um conteúdo desinformativo e acredita no que está recebendo, há um prejuízo potencial tanto para a saúde de um indivíduo, quanto para as pessoas que estão à sua volta, e ainda para a coletividade como um todo. Em um momento de pandemia e infodemia, pode resultar em mortes. De acordo com Kuzmanovic apud Zarocostas (2020, tradução nossa), “o que está em jogo durante um surto é garantir que as pessoas façam a coisa certa para controlar a doença ou para mitigar seu impacto”. 22 Nesse contexto, pode-se considerar a postura do YouTube como um aspecto potencialmente positivo no enfrentamento da pandemia, mas é preciso notar que há outros meios e dinâmicas para a circulação de desinformações

\section{Referências}

ALEXANDER, J. YouTube is demonetizing videos about coronavirus, and creators are mad. The Verge, 4 mar 2020.4 Disponível <https://www.theverge.com/2020/3/4/21164553/youtube-coronavirus-demonetizationsensitive-subjects-advertising-guidelines-revenue>. Acesso em: 9 jun. 2020.

AZEVEDO, R. Youtube remove vídeo em que Olavo de Carvalho diz que coronavírus não mata. Reinaldo Azevedo, 23 mar 2020. Disponível em: <https://noticias.uol.com.br/colunas/reinaldo-azevedo/2020/03/23/youtube-removepost-em-que-olavo-de-carvalho-diz-que-coronavirus-nao-existe.htm>. Acesso em: 2 jul. 2020.

BURGESS, J.; GREEN, J. YouTube: Online video and participatory culture. [s.l.] John Wiley \& Sons, 2018.

D’ANDRÉA, C. Pesquisando plataformas online: conceitos e métodos. Salvador: EDUFBA, 2020.

FERNANDES, C. M. et al. A rede de desinformação e a saúde em risco: uma análise das fake news contidas em "As 10 razões pelas quais você não deve vacinar seu filho". Revista Eletrônica de Comunicação, Informação e Inovação em Saúde, v. 14, n. 2, 26 jun. 2020.

GILLESPIE, T. Custodians of the Internet: Platforms, content moderation, and the hidden decisions that shape social media. [s.l.] Yale University Press, 2018.

22 No original: "What is at stake during an outbreak is making sure people will do the right thing to control the disease or to mitigate its impact." 
GOOGLE. Como ganhar dinheiro no YouTube. Ajuda do AdSense, 2020. Disponível em: <https://support.google.com/adsense/answer/72857>. Acesso em: 20 jul. 2020.

GOOGLE. Trazendo mais transparência e contexto para o YouTube no Brasil. O blog do Google Brasil, 26 nov 2019. Disponível em: <https: / /brasil.googleblog.com/2019/11/trazendo-mais-transparencia-e-contexto.html> Acesso em: 23 jul. 2020.

LEMOS, V. Os polêmicos vídeos de médicos que recomendam tratamentos sem comprovação para covid-19. BBC News Brasil, 15 jul 2020. Disponível em: <https://www.bbc.com/portuguese/brasil-53377938>. Acesso em: 19 jul. 2020.

LI, H. O.-Y. et al. YouTube as a source of information on COVID-19: a pandemic of misinformation? BMJ Global Health, v. 5, n. 5, p. e002604, 1 maio 2020.

MCAWEENEY, E. Who Benefits from Health Misinformation? Data \& Society: Points, 30 mar 2020. Disponível em: <https://points.datasociety.net/who-benefits-from-healthmisinformation-8d094804058d>. Acesso em: 30 mar. 2020.

MINISTÉRIO DA SAÚDE. Brasil confirma primeiro caso da doença. Ministério da Saúde, 27 fev 2020. Disponível em: <https://www.saude.gov.br/noticias/agencia-saude/46435brasil-confirma-primeiro-caso-de-novo-coronavirus>. Acesso em: 8 jul. 2020. 2020.

NEWMAN, N. et al. Reuters Institute Digital News Report 2019. Reuters Institute for the Study of Journalism, 2019.2 Disponivel em: <https://reutersinstitute.politics.ox.ac.uk/sites/default/files/inline-

files/DNR_2019_FINAL.pdf>. Acesso em: 29 mar. 2020.

OHLHEISER, A. How covid-19 conspiracy theorists are exploiting YouTube culture. MIT Technology Review, 7 mai. 2020a Disponível em: <https://www.technologyreview.com/2020/05/07/1001252/youtube-covid-conspiracytheories/>. Acesso em: 8 maio de 2020.

OHLHEISER, A. How covid-19 conspiracy videos keep getting millions of views. MIT Technology Review, 28 jul. 2020b. Disponível em: <https: / /www.technologyreview.com/2020/07/28/1005738/hydroxychloroquine-covid-19misinformation-video/>. Acesso em: 2 ago 2020.

PEREZ, S. YouTube will now allow creators to monetize videos about coronavirus and COVID-19. Techcrunch, 11 mar 2020. Disponível em: <https://social.techcrunch.com/2020/03/11/youtube-will-now-allow-creators-tomonetize-videos-about-coronavirus-and-covid-19/>. Acesso em: 19 jul. 2020. 
PLANTIN, J. et al. Infrastructure studies meet platform studies in the age of Google and Facebook. New Media \& Society. v.4. p.1-18, 2016

RIEDER, B.; MATAMOROS-FERNÁNDEZ, A.; COROMINA, Ò. From ranking algorithms to 'ranking cultures': Investigating the modulation of visibility in YouTube search results. Convergence, v. 24, n. 1, p. 50-68, 10 jan. 2018.

ROBERTS, S. T. Over*Flow: Digital Humanity: Social Media Content Moderation and the Global Tech Workforce in the COVID-19 Era. Flow, 19 mar 2020. Disponível em: <https://www.flowjournal.org/2020/03/digital-humanity/>. Acesso em: 21 jul. 2020.

SILVA, V. H. YouTube remove teorias de conspiração sobre COVID-19 e 5G. Tecnoblog, 6 abr 2020. Disponível em: <https://tecnoblog.net/332812/youtube-remove-teoriasconspiracao-covid-19-5g/>. Acesso em: 2 jul. 2020.

STATISTA. Most used social media platform. Statista, 24 de julho de 2020. Disponível em: <https://www.statista.com/statistics/272014/global-social-networks-ranked-by-numberof-users/>. Acesso em: 9 jul. 2020.

TILT. YouTube exclui canal de teórico da conspiração com vídeos contrários à OMS. Tilt, 4 mai 2020. Disponível em: <https://www.uol.com.br/tilt/noticias/redacao/2020/05/04/youtube-exclui-canalcontrarios-oms.htm>. Acesso em: 2 jul. 2020.

VENTURA, F. Google remove vídeos alegando "farsa do coronavírus" em SP. Tecnoblog, 11 mai 2020. Disponível em: <https://tecnoblog.net/337988/google-remove-videos-alegandofarsa-do-coronavirus-em-sp/>. Acesso em: 2 jul. 2020.

VENTURINI, T. et al. A reality check(list) for digital methods. New Media \& Society, v. 20, n. 11, p. 4195-4217, 1 nov. 2018.

VOSOUGHI, S.; ROY, D.; ARAL, S. The spread of true and false news online. Science, v. 359, n. 6380, p. 1146-1151, 2018.

WARDLE, C.; DERAKHSHAN, H. Information Disorder: Toward an interdisciplinary framework for research and policymaking. Council of Europe report, DGI (2017), v. 9, 2017.

YOUTUBE. Atualização sobre a monetização no conteúdo relacionado à COVID-19. Ajuda do YouTube, 2020a. Disponível em: <https: / / support.google.com/youtube/answer/9803260?hl=pt-BR\&ref_topic=6151248>. Acesso em: 30 jun. 2020. 
YOUTUBE. Atualizações sobre o coronavírus 2019 (COVID-19). Ajuda do Youtube, 2020b. Disponível em: <https://support.google.com/youtube/answer/9777243?hl=pt-BR>. Acesso em: 29 jun. 2020.

YOUTUBE. Coronavirus: An update on creator support and resources. YouTube Creator Blog, 11 mar 2020c. Disponível em: <https://youtubecreators.googleblog.com/2020/03/coronavirus-update-on-creator-support.html>. Acesso em: 9 jun. 2020.

YOUTUBE. Diretrizes de conteúdo adequado para publicidade. Ajuda do Youtube, 2020d. Disponível em: <https://support.google.com/youtube/answer/6162278?hl=pt-BR>. Acesso em: 9 jun. 2020.

YOUTUBE. Expanding fact checks on YouTube to the United States. Official YouTube Blog, 28 abr 2020e. Disponível em: <https://youtube.googleblog.com/2020/04/expanding-factchecks-on-youtube-to-united-states.html>. Acesso em: 23 jul. 2020.

YOUTUBE. Política de conteúdo perigoso ou nocivo. Ajuda do Youtube, 2020f. Disponível em: <https://support.google.com/youtube/answer/2801964>. Acesso em: 9 jun. 2020.

YOUTUBE. Política de informações médicas incorretas relacionadas à COVID-19. Ajuda do YouTube, 2020g. Disponível em: <https://support.google.com/youtube/answer/9891785>. Acesso em: 2 jul.

YOUTUBE. Termos de serviço. YouTube, 2019a. Disponível em: <https://www.youtube.com/t/terms?archive=20180525>. Acesso em: 13 jul. 2020.

YOUTUBE. Termos de serviço. YouTube, 2019b. Disponível em: <https://www.youtube.com/static?gl=BR\&template=terms\&hl=pt>. Acesso em: 13 jul. 2020.

YOUTUBE. The Four Rs of Responsibility, Part 2: Raising authoritative content and reducing borderline content and harmful misinformation. YouTube Official Blog, 02 dez 2019c. Disponível em: <https://youtube.googleblog.com/2019/12/the-four-rs-of-responsibilityraise-and-reduce.html>. Acesso em: 20 abr. 2020.

YOUTUBE. Update for Creators on COVID-19 \& the Impact on our Review Teams. YouTube Help, 16 mar 2020h. Disponível em: <https://support.google.com/youtube/thread/33835276?hl=en>. Acesso em: 30 jun. 2020.

YOUTUBE. Vista geral da autocertificação do YouTube. YouTube Ajuda, 2020j. Disponível em: <https://support.google.com/youtube/answer/7687980?hl=pt>. Acesso em: 30 jun. 2020. 
ZAROCOSTAS, J. How to fight an infodemic. The Lancet, v. 395, n. 10225, p. 676, 29 fev. 2020. 
Como citar este artigo

FONSECA, Gregório de Almeida; D’ANDRÉA, Carlos Frederico de Brito. Governança e mediações algorítmicas da plataforma YouTube durante a pandemia de Covid-19. Revista Dispositiva. [on-line] Disponível em: <http://periodicos.pucminas.br/index.php/dis positiva> Dossiê: Comunicação, política e saúde. Editoras Responsáveis: Fernanda Sanglard e Vanessa Veiga de Oliveira. Volume 9, Número 16, Belo Horizonte, dezembro de 2020, p. 6-26. Acesso em “dia/mês/ano".

Texto recebido em: 05/08/2020

Texto aprovado em: 10/12/2020 